\title{
Mutual Motion Camouflage in 3D*
}

\author{
M. Mischiati P. S. Krishnaprasad \\ Institute for Systems Research and Department of Electrical and \\ Computer Engineering, University of Maryland, College Park, MD \\ 20742, USA (email: mismat@umd.edu, krishna@umd.edu)
}

\begin{abstract}
Motion camouflage is a type of pursuit strategy observed in nature. In this paper, we show that when a pair of autonomous agents, modeled as particles in three dimensions, engage in mutual motion camouflage, their dynamically coupled trajectories exhibit geometrically interesting structure, of possible relevance to applications such as surveillance.
\end{abstract}

\section{INTRODUCTION}

Pursuit and evasion phenomena play an important role not only in human society (e.g. in warfare and sports) but also and foremost in the animal world. For many species, survival and reproduction depend on their capabilities of effectively pursuing prey or mates and evading predators. Pursuit phenomena may also contribute to the generation of collective motion in homogeneous groups of animals, as each individual may tend to move closer to other members to avoid predation [Hamilton, 1971].

The Motion Camouflage(MC), or Constant Absolute Target Direction(CATD), pursuit strategy has been observed in the behavior of several animal species. We say that a pursuer is using this strategy if it approaches its target while maintaining constant absolute direction of the imaginary baseline connecting the two individuals. From Justh and Krishnaprasad [2006], if $\mathbf{r}$ is the relative position of the evader with respect to the pursuer, and $\dot{\mathbf{r}}$ is its time derivative, then the motion camouflage pursuit strategy holds when:

$$
\Gamma(\mathbf{r}, \dot{\mathbf{r}}) \triangleq \frac{\dot{\mathbf{r}}}{|\dot{\mathbf{r}}|} \cdot \frac{\mathbf{r}}{|\mathbf{r}|}=-1 .
$$

Many insects which navigate using vision have compound eyes that are effective at detecting motion transverse to the field of view (i.e. optic flow), but limited stereoscopic vision entails poor sensitivity to looming. Strategy (1) is very apt in this setting since it enables the pursuer to camouflage its motion by nulling the optic flow it produces in the visual field of the target. Empirical evidence in Srinivasan and Davey [1995] and Mizutani et al. [2003] suggests that the motion camouflage strategy is employed by male hoverflies and dragonflies for both mating and territorial battles; the same references also present evidence for a variant of (1) which allows the pursuer to appear stationary relative to a specific background feature in the visual field of the evader. The motion camouflage pursuit strategy is also geometrically identical to the one used by echolocating bats in chasing insect prey, the "constant absolute target direction strategy" in Ghose et al. [2006]. The biological

\footnotetext{
* This research was supported in part by the Air Force Office of Scientific Research under AFOSR grant FA95501010250; by the Army Research Office under ARO grant W911NF0610325; by the ODDR\&E MURI2007 Program Grant N000140710734 (through the Office of Naval Research).
}

reason for this is clearly different from that of visual insects, and seems to be rooted in the properties of timeoptimality of this strategy for the capture of evaders moving in unpredictable fashion. The superiority of the motion camouflage strategy with respect to alternative pursuit strategies is studied from a game-theoretic point of view in Wei et al. [2009].

Inspired by the biological relevance of motion camouflage pursuit, we have introduced in a recent paper [Mischiati and Krishnaprasad, 2011] a simple mathematical model which highlights its potential use as a building-block for obtaining coordinated motion. In this model, called Mutual Motion Camouflage(MMC), two individuals are in mutual pursuit with a constant-speed, planar implementation of motion camouflage (see Justh and Krishnaprasad [2006]). The resulting choreography produces trajectories that have interesting region-filling properties. In Mischiati and Krishnaprasad [2010], mutual motion camouflage was used to design control laws and initialization rules for a pair of artificial agents moving in $\mathbb{R}^{2}$ to achieve dense coverage (monitoring) of a region of the plane.

In this paper, we extend this research program to the three-dimensional setting. Using a biologically plausible implementation of the motion camouflage pursuit strategy in $\mathbb{R}^{3}$ (first presented in Reddy et al. [2006]), we introduce a three-dimensional model for mutual motion camouflage. We show that the resulting highly coordinated trajectories correspond to "periodically modulated" helices. The characteristic region-filling properties of planar mutual motion camouflage can be recovered by a suitable projection. We moreover propose a new set of control laws and initialization strategies that would allow a pair of autonomous agents moving in $\mathbb{R}^{3}$ (e.g. UAVs) to perform surveillance tasks that extend those presented in Mischiati and Krishnaprasad [2010], for example by allowing freedom of choice of the plane on which the monitored region lies.

We begin by recalling in section 2 the definition of mutual motion camouflage in $\mathbb{R}^{2}$ and results from Mischiati and Krishnaprasad [2010, 2011]. We then proceed to state the mutual motion camouflage feedback laws in section 3 , providing a closed loop analysis in section 4, and applications to coverage in section 5 . 


\section{MUTUAL MOTION CAMOUFLAGE IN $\mathbb{R}^{2}$}

Let each agent be modeled as a unit-mass particle moving at constant speed in $\mathbb{R}^{2}$. Then the motion of the $i$-th agent $(i=1,2)$ can be described by the (planar) natural Frenet frame equations [Bishop, 1975]:

$$
\begin{aligned}
\dot{\mathbf{r}_{\mathbf{i}}} & =\nu_{i} \mathbf{x}_{\mathbf{i}} \\
\dot{\mathbf{x}_{\mathbf{i}}} & =\nu_{i} u_{i} \mathbf{y}_{\mathbf{i}} \\
\dot{\mathbf{y}_{\mathbf{i}}} & =-\nu_{i} u_{i} \mathbf{x}_{\mathbf{i}} .
\end{aligned}
$$

Here $\mathbf{y}_{\mathbf{i}} \triangleq \mathbf{x}_{\mathbf{i}}{ }^{\perp}$ is the counterclockwise rotation of $\mathbf{x}_{\mathbf{i}}$ by $\pi / 2$ radians, and completes with $\mathbf{x}_{\mathbf{i}}$ an orthonormal frame at $\mathbf{r}_{\mathbf{i}}$ attached to the curve traced by the $i$-th particle (assumed twice-differentiable). For each agent, the control is the curvature (steering) $u_{i}$, which leaves unchanged the speed $\nu_{i}$.

A biologically-plausible curvature control law for agent $i$ to implement the motion camouflage strategy is (from Justh and Krishnaprasad [2006]):

$$
u_{i}=-\mu\left(\frac{\mathbf{r}}{|\mathbf{r}|} \cdot \dot{\mathbf{r}}^{\perp}\right),
$$

where $\mu>0$ is a constant gain and $\mathbf{r}$ is the relative position of the evader with respect to agent $i$. Provided that the initial conditions are such that $\Gamma \neq 1$ and $|\mathbf{r}(0)|>0$, and the evader moves slower than the pursuer along trajectories with bounded and continuous curvature, (3) guarantees that the motion camouflage state (1) is accessible in finite time [Justh and Krishnaprasad, 2006].

Definition 1. The Mutual Motion Camouflage (MMC) planar model describes the case in which two agents, subject to dynamics (2), are in mutual pursuit with the motion camouflage feedback law (3) and gains inversely proportional to the speeds (to preserve symmetry even when the individual speeds are different):

$$
u_{1} \nu_{1}=u_{2} \nu_{2}=-\mu \lambda, \lambda \triangleq\left(\frac{\mathbf{r}}{|\mathbf{r}|} \cdot \dot{\mathbf{r}}^{\perp}\right), \mathbf{r} \triangleq \mathbf{r}_{1}-\mathbf{r}_{\mathbf{2}} .
$$

The closed loop dynamics of mutual motion camouflage, expressed in terms of the relative position $\mathbf{r}$ and the scaled center of mass position $\mathbf{z} \triangleq \mathbf{r}_{\mathbf{1}}+\mathbf{r}_{\mathbf{2}}$, are:

$$
\begin{aligned}
& \ddot{\mathbf{r}}=-\mu \lambda \dot{\mathbf{r}}^{\perp}=\mu\left[\left(\frac{\mathbf{r}}{|\mathbf{r}|} \cdot \dot{\mathbf{r}}\right) \dot{\mathbf{r}}-|\dot{\mathbf{r}}|^{2} \frac{\mathbf{r}}{|\mathbf{r}|}\right] \\
& \ddot{\mathbf{z}}=-\mu \lambda \dot{\mathbf{z}}^{\perp},
\end{aligned}
$$

where in (5) we have used the decomposition of $\mathbf{r} /|\mathbf{r}|$ with respect to the orthonormal basis composed of $\dot{\mathbf{r}} /|\dot{\mathbf{r}}|$ and $\dot{\mathbf{r}}^{\perp} /|\dot{\mathbf{r}}|$.

The main results on the analysis of (5)-(6) can be summarized as follows ${ }^{1}$ [Mischiati and Krishnaprasad, 2010, 2011]:

(a) The relative distance between the agents, $\rho \triangleq|\mathbf{r}|$, is subject to Lagrangian second-order dynamics $\ddot{\rho}=(1 / \rho-$ $\mu)\left(\delta^{2}-\dot{\rho}^{2}\right)$, where $\delta \triangleq|\dot{\mathbf{r}}|$ is constant. The orbits $(\rho(t)$, $\dot{\rho}(t))$ are periodic and are level sets of the energy function $E(\rho, \dot{\rho})=\rho^{2}\left(\delta^{2}-\dot{\rho}^{2}\right) e^{-2 \mu \rho}$. A special case is the equilibrium point $\rho(t)=1 / \mu, \dot{\rho}(t)=0$ corresponding to circular relative motion.

1 This summary describes the behavior of the system for generic initial conditions. There is however a thin set of special initial conditions yielding different results; for example there are (nonattracting) invariant manifolds characterized by $\Gamma(\mathbf{r}, \dot{\mathbf{r}})= \pm 1$. (b) The relative position $\mathbf{r}(t)$ varies instead aperiodically (and its dynamics (5) are not Lagrangian) filling in time an annular region of inner and outer radii $\rho_{\min }>0$ and $\rho_{\max }>\rho_{\min }$ given by the solutions to the equation: $\rho^{2} \delta^{2} e^{-2 \mu \rho}=E\left(\rho_{0}, \dot{\rho}_{0}\right)$.

(c) There exists a fixed vector $\mathbf{z}_{\mathbf{0}} \in \mathbb{R}^{2}$, which depends only on the initial conditions, such that $\zeta \triangleq\left|\mathbf{z}-\mathbf{z}_{\mathbf{0}}\right|$ evolves (periodically) according to $\zeta(t)=\theta \rho(t) / \delta \forall t$, where $\theta \triangleq|\dot{\mathbf{z}}|$ is constant.

(d) The center of mass trajectory $\mathbf{z}(t) / 2$, and similarly the individual particle trajectories $\mathbf{r}_{\mathbf{1}}(t)=(\mathbf{z}(t)+\mathbf{r}(t)) / 2$ and $\mathbf{r}_{\mathbf{2}}(t)=(\mathbf{z}(t)-\mathbf{r}(t)) / 2$, fill in time annular regions centered at $\mathbf{z}_{\mathbf{0}} / 2$ and having inner and outer radii proportional to $\rho_{\min }, \rho_{\max }$.

(e) The closed-loop dynamics of the scalar variable $\lambda$ ("optic flow") can be entirely expressed in terms of the distance $\rho$ (and initial conditions):

$$
\lambda(t)=\lambda_{0} \rho_{0} e^{\mu\left(\rho(t)-\rho_{0}\right)} / \rho(t)
$$

If the control laws (4) are modified as follows:

$$
u_{1} \nu_{1}=u_{2} \nu_{2}=-\mu \lambda+k_{d} \lambda \dot{\rho}\left(E(\rho, \dot{\rho})-E_{d}\right), \quad k_{d}>0,
$$

then (almost) all of the orbits $(\rho(t), \dot{\rho}(t))$ are attracted to the periodic orbit associated to a prescribed energy level $E_{d}$. With surveillance applications in mind, we have exploited in Mischiati and Krishnaprasad [2010] the resulting "modified (planar) MMC model" to produce design parameters (choices of $\mu, E_{d}$ and initial conditions) that enable a chosen annular region to be covered (monitored) by the agents.

\section{THREE-DIMENSIONAL MODEL OF MMC}

We extend the dynamical model used in the planar case, by assuming that each individual is a unit-mass particle moving at constant speed along twice-differentiable curves in $\mathbb{R}^{3}$. Because of the constant speed constraint, the only control authority for each particle is on the direction of motion. In mechanical terms, this corresponds to the particles being subject to gyroscopic forces, which preserve their total kinetic energies. The dynamics of each agent $(i=1,2)$ can be conveniently described using the natural Frenet framing of curves in $\mathbb{R}^{3}$ (as in Bishop [1975], Justh and Krishnaprasad [2005]):

$$
\begin{aligned}
& \dot{\mathbf{r}_{\mathbf{i}}}=\nu_{i} \mathbf{x}_{\mathbf{i}} \\
& \dot{\mathbf{x}_{\mathbf{i}}}=\nu_{i}\left(u_{i} \mathbf{y}_{\mathbf{i}}+v_{i} \mathbf{z}_{\mathbf{i}}\right) \\
& \dot{\mathbf{y}_{\mathbf{i}}}=-\nu_{i} u_{i} \mathbf{x}_{\mathbf{i}} \\
& \dot{\mathbf{z}_{\mathbf{i}}}=-\nu_{i} v_{i} \mathbf{x}_{\mathbf{i}} .
\end{aligned}
$$

Here $\mathbf{x}_{\mathbf{i}}$ is the unit vector in the direction of motion of the $i$-th particle, while $\mathbf{y}_{\mathbf{i}}, \mathbf{z}_{\mathbf{i}}$ complete with $\mathbf{x}_{\mathbf{i}}$ a right-handed orthonormal frame for the particle trajectory. Notice that once an initial choice of $\mathbf{y}_{\mathbf{i}}(0), \mathbf{z}_{\mathbf{i}}(0)$ is made, the frame evolution with time is well-defined by (9). The natural curvatures $u_{i}, v_{i}$ can be thought of as the controls, which preserve the speed $\nu_{i}$; to see this, notice that they act on $\dot{\mathbf{x}}_{\mathbf{i}}$ in directions $\mathbf{y}_{\mathbf{i}}$ and $\mathbf{z}_{\mathbf{i}}$, orthogonal to $\mathbf{x}_{\mathbf{i}}$.

Biologically-inspired curvature control laws that implement the motion camouflage pursuit strategy in $\mathbb{R}^{3}$ were introduced in Reddy et al. [2006]; for a particle $i$ pursuing particle $j$ (the evader), they are: 
$u_{i}=-\mu\left[\mathbf{z}_{\mathbf{i}} \cdot\left(\dot{\mathbf{r}}_{\mathbf{i}, \mathbf{j}} \times \frac{\mathbf{r}_{\mathbf{i}, \mathbf{j}}}{\left|\mathbf{r}_{\mathbf{i}, \mathbf{j}}\right|}\right)\right], v_{i}=\mu\left[\mathbf{y}_{\mathbf{i}} \cdot\left(\dot{\mathbf{r}}_{\mathbf{i}, \mathbf{j}} \times \frac{\mathbf{r}_{\mathbf{i}, \mathbf{j}}}{\left|\mathbf{r}_{\mathbf{i}, \mathbf{j}}\right|}\right)\right]$,

where $\mu>0$ is a constant gain, and $\mathbf{r}_{\mathbf{i}, \mathbf{j}}=\mathbf{r}_{\mathbf{i}}-\mathbf{r}_{\mathbf{j}}$.

Just as in the planar case, if the initial conditions satisfy $\Gamma \neq 1$ and $|\mathbf{r}(0)|>0$, and the motion of the evader satisfies certain constraints (slower than the pursuer, trajectories with bounded and continuous curvatures), then (10) guarantees that the motion camouflage state is accessible in finite time [Reddy et al., 2006]. This is guaranteed even if the speeds of the two particles vary with time, provided that the speed profiles are bounded and have bounded and piecewise continuous derivatives. In support to the claim that these control laws are biologically feasible, Reddy [2007] reports high correlation between the curvatures produced by a delayed version of (10), which accounts for sensorimotor reaction times, and the actual trajectory curvatures extracted from experimental flight data of bats engaged in prey pursuit.

As in (4), for three-dimensional definition of mutual motion camouflage we require both particles to apply the motion camouflage control laws (10) scaled by the reciprocals of their speeds.

Definition 2. We define as Mutual Motion Camouflage in 3D (MMC-3D) the system composed of two particles $(i=1,2)$ moving in $\mathbb{R}^{3}$ with dynamics subject to $(9)$ and the following motion camouflage curvature laws (here $\left.\mathbf{r} \triangleq \mathbf{r}_{1}-\mathbf{r}_{2}\right)$ :

$$
u_{i}=-\frac{\mu}{\nu_{i}}\left[\mathbf{z}_{\mathbf{i}} \cdot\left(\dot{\mathbf{r}} \times \frac{\mathbf{r}}{|\mathbf{r}|}\right)\right], v_{i}=\frac{\mu}{\nu_{i}}\left[\mathbf{y}_{\mathbf{i}} \cdot\left(\dot{\mathbf{r}} \times \frac{\mathbf{r}}{|\mathbf{r}|}\right)\right]
$$

Remark 1. While the control laws for mutual motion camouflage in the plane satisfy $u_{1} \nu_{1}=u_{2} \nu_{2}$, the same is not true for (11) (which neither satisfy $v_{1} \nu_{1}=v_{2} \nu_{2}$ ). The control laws for MMC-3D depend on the axes $\mathbf{y}_{\mathbf{i}}, \mathbf{z}_{\mathbf{i}}$, which are in general different for each particle. The curvature laws (11) do not even satisfy the symmetry condition $\kappa_{1} \nu_{1}=\kappa_{2} \nu_{2}$ on the "total" curvatures $\kappa_{i} \triangleq\left|\ddot{\mathbf{r}_{\mathbf{i}}}\right| / \nu^{2}=$ $\sqrt{u_{i}^{2}+v_{i}^{2}}$, which are independent of the initial choice of frame. Nevertheless it is clear from the following analysis that the choice (11) truly yields dynamics that are a natural extension of MMC to $\mathbb{R}^{3}$.

\section{ANALYSIS OF MMC IN $\mathbb{R}^{3}$}

The study of mutual motion camouflage in three dimensions involves the analysis of the closed-loop system obtained by substituting (11) in the particle dynamics (9). The closed-loop system can be conveniently expressed via two sets of differential equations: one describing the relative motion between the particles and one describing the center of mass motion. Introducing $\mathbf{r}=\mathbf{r}_{1}-\mathbf{r}_{\mathbf{2}}$, $\mathbf{g}=\nu_{1} \mathbf{x}_{\mathbf{1}}-\nu_{2} \mathbf{x}_{\mathbf{2}}, \mathbf{z}=\mathbf{r}_{\mathbf{1}}+\mathbf{r}_{\mathbf{2}}$ and $\mathbf{k}=\nu_{1} \mathbf{x}_{\mathbf{1}}+\nu_{2} \mathbf{x}_{\mathbf{2}}$, these are:

$$
\begin{aligned}
& \dot{\mathbf{r}}=\mathbf{g} \\
& \dot{\mathbf{g}}=\mu\left[\mathrm{g} \times\left(\mathrm{g} \times \frac{\mathbf{r}}{|\mathbf{r}|}\right)\right], \\
& \dot{\mathbf{z}}=\mathbf{k} \\
& \dot{\mathbf{k}}=\mu\left[\mathbf{k} \times\left(\mathbf{g} \times \frac{\mathbf{r}}{|\mathbf{r}|}\right)\right] .
\end{aligned}
$$

A vector quantity that plays an important role in the analysis of both the relative motion and the center of mass motion is the relative angular momentum $\mathbf{l} \triangleq \mathbf{r} \times \mathbf{g}$, which evolves according to the following dynamics:

$$
\mathrm{i}=\mu \gamma \mathbf{l}
$$

where $\gamma \triangleq \mathbf{g} \cdot \mathbf{r} /|\mathbf{r}|=\dot{\rho}, \rho \triangleq|\mathbf{r}|$. Hence the direction of 1 remains constant in time (it depends only on the initial conditions). In the remainder of the paper, we will denote as $\mathbf{e}_{\mathbf{l}}$ the constant unit vector in the direction of $\mathbf{l}(t)$, and $l(t)$ the time-varying magnitude of $\mathbf{l}(t)$, which is given by:

$$
l(t)=l(0) e^{\int_{0}^{t} \mu \gamma(s) d s}=l(0) e^{\mu(\rho(t)-\rho(0))} .
$$

We will exploit this result on the behavior of the relative angular momentum to derive the trajectories induced by MMC-3D in three steps: derivation of the relative motion between the agents, derivation of the center of mass trajectory, and finally reconstruction of the individual trajectories.

\subsection{Relative motion between the agents}

Using the triple vector product rule, the system of equations (12) can be expressed in a form identical to (5):

$$
\ddot{\mathbf{r}}=\dot{\mathrm{g}}=\mu\left[\left(\frac{\mathbf{r}}{|\mathbf{r}|} \cdot \dot{\mathbf{r}}\right) \dot{\mathbf{r}}-|\dot{\mathbf{r}}|^{2} \frac{\mathbf{r}}{|\mathbf{r}|}\right] \text {. }
$$

Of course the difference is that here $\mathbf{r} \in \mathbb{R}^{3}$, while in (5) $\mathbf{r} \in \mathbb{R}^{2}$. Nevertheless, by (12), $\ddot{\mathbf{r}}=-\mu(\dot{\mathbf{r}} \times \mathbf{l}) / \rho$ is always orthogonal to $\mathbf{e}_{\mathbf{l}}$. Since the vectors $\mathbf{r}$ and $\dot{\mathbf{r}}$ are orthogonal to $\mathbf{e}_{\mathbf{l}}$ (by the definition of $\mathbf{l}$ ), we have that the component of $\mathbf{r}$ in the direction $\mathbf{e}_{\mathbf{l}}$ is always zero. Hence the projection of (16) onto the plane (through the origin) orthogonal to $\mathbf{e}_{\mathbf{l}}$ is identical to the relative motion dynamics in planar MMC (5); we conclude that the relative position $\mathbf{r}(t)$ fills annular regions on the plane orthogonal to $\mathbf{e}_{\mathbf{l}}$, with the relative distance $\rho(t)$ oscillating periodically between extrema $\rho_{\min }$ and $\rho_{\max }$. This also implies that the magnitude $l(t)$ of the relative angular momentum, given by (15), varies periodically.

\subsection{Center of mass motion}

The (scaled) center of mass velocity $\mathbf{k} \triangleq \dot{\mathbf{z}}$ is subject to the linear time-varying dynamics (13): $\dot{\mathbf{k}}(t)=\mu(\mathbf{l}(t) \times$ $\mathbf{k}(t)) / \rho(t)=\mathrm{A}(t) \mathbf{k}(t)$, with $\mathrm{A}(t) \triangleq \mu(l(t) / \rho(t)) \hat{\mathbf{e}}_{\mathbf{l}}, \hat{\mathbf{e}}_{\mathbf{l}} \in$ so(3) being the skew-symmetric matrix associated to the unit vector $\mathbf{e}_{\mathbf{l}}$. The solution of this system is:

$$
\mathbf{k}(t)=\exp \left(\int_{0}^{t} \mathrm{~A}(\sigma) d \sigma\right) \mathbf{k}(0)=\exp \left(\int_{0}^{t} \mu \frac{l(\sigma)}{\rho(\sigma)} d \sigma \hat{\mathbf{e}}_{\mathbf{l}}\right) \mathbf{k}(0) .
$$

From the properties of the exponential map (exp : so $(3) \rightarrow$ $\mathrm{SO}(3))$ :

$$
\mathbf{k}(t)=\operatorname{Rot}\left(\mathbf{e}_{\mathbf{l}}, \alpha(t)\right) \mathbf{k}(0),
$$

where $\alpha(t) \triangleq \mu \int_{0}^{t} \frac{l(\sigma)}{\rho(\sigma)} d \sigma$. The notation $\operatorname{Rot}(\mathbf{e}, \alpha) \in \operatorname{SO}(3)$ denotes the matrix corresponding to counterclockwise rotation by an angle $\alpha$ about the axis $\mathbf{e} \in \mathbb{R}^{3}$.

Remark 2. Since $\rho(t)>0 \forall t$ and $l(t) \geq 0 \forall t$, the "rotation angle" satisfies $\alpha(t) \geq 0 \forall t, \alpha(t)=0 \Leftrightarrow l(0)=0$. Hence the center of mass velocity is constant in time only if either $\mathbf{r}(0)$ is parallel to $\mathbf{g}(0)$ (so that $l(0)=0$ ) or $\mathbf{k}(0)$ is parallel 
to $\mathbf{e}_{\mathbf{l}}=\mathbf{l}(0) / l(0)$. On the other hand, the magnitude of $\mathbf{k}$, and hence the center of mass speed, is always constant in time (just as in the planar case); we call this quantity $\theta$.

The rotation matrix in (17) can be expanded using Rodrigues formula, yielding the more explicit expression:

$$
\begin{aligned}
\mathbf{k}(t)= & \cos (\alpha(t)) \mathbf{k}(0)+\sin (\alpha(t))\left(\mathbf{e}_{\mathbf{l}} \times \mathbf{k}(0)\right) \\
& +(1-\cos (\alpha(t)))\left(\mathbf{k}(0) \cdot \mathbf{e}_{\mathbf{l}}\right) \mathbf{e}_{\mathbf{l}} .
\end{aligned}
$$

Equation (18) highlights the components of the center of mass velocity in three fixed (but in general not orthogonal) directions: $\mathbf{k}(0), \mathbf{e}_{\mathbf{l}}$ and $\mathbf{e}_{\mathbf{l}} \times \mathbf{k}(0)$. Provided that $\mathbf{e}_{\mathbf{l}} \times$ $\mathbf{k}(0) \neq 0$ (if this is not the case, the center of mass velocity is constant as explained in remark 2), it is possible to conveniently rewrite (18) in terms of an orthonormal frame $\left(\mathbf{e}_{\mathbf{x}}, \mathbf{e}_{\mathbf{y}}, \mathbf{e}_{\mathbf{l}}\right)$ in $\mathbb{R}^{3}$, defined by $\mathbf{e}_{\mathbf{x}} \triangleq \mathbf{e}_{\mathbf{l}} \times \mathbf{k}(0) /\left|\mathbf{e}_{\mathbf{l}} \times \mathbf{k}(0)\right|$ and $\mathbf{e}_{\mathbf{y}} \triangleq \mathbf{e}_{\mathbf{l}} \times \mathbf{e}_{\mathbf{x}}=\mathbf{e}_{\mathbf{l}} \times\left(\mathbf{e}_{\mathbf{l}} \times \mathbf{k}(0)\right) /\left|\mathbf{e}_{\mathbf{l}} \times \mathbf{k}(0)\right|=\left(\mathbf{e}_{\mathbf{l}}(\mathbf{k}(0)\right.$. $\left.\left.\mathbf{e}_{\mathbf{l}}\right)-\mathbf{k}(0)\right) /\left|\mathbf{e}_{\mathbf{l}} \times \mathbf{k}(0)\right|$. It is a simple algebraic exercise to show that the resulting equation can be expressed as:

$$
\begin{aligned}
\mathbf{k}(t)= & -\left|\mathbf{e}_{\mathbf{l}} \times \mathbf{k}(0)\right| \sin (-\alpha(t)) \mathbf{e}_{\mathbf{x}} \\
& -\left|\mathbf{e}_{\mathbf{l}} \times \mathbf{k}(0)\right| \cos (-\alpha(t)) \mathbf{e}_{\mathbf{y}}+\left(\mathbf{k}(0) \cdot \mathbf{e}_{\mathbf{l}}\right) \mathbf{e}_{\mathbf{l}},
\end{aligned}
$$

or component-wise:

$$
\mathbf{k}(t)=\left[\begin{array}{c}
k_{x}(t) \\
k_{y}(t) \\
k_{l}(t)
\end{array}\right]=\left[\begin{array}{c}
-\left|\mathbf{e}_{\mathbf{l}} \times \mathbf{k}(0)\right| \sin (-\alpha(t)) \\
-\left|\mathbf{e}_{\mathbf{l}} \times \mathbf{k}(0)\right| \cos (-\alpha(t)) \\
\mathbf{k}(0) \cdot \mathbf{e}_{\mathbf{l}}
\end{array}\right] .
$$

From (20), it is clear that the center of mass velocity in the direction of $\mathbf{e}_{\mathbf{l}}$ is constant, hence the component $z_{l}(t)$ of the (scaled) center of mass in that direction grows linearly. On the other hand, it is possible to prove that the projection of $\mathbf{z}$ on the plane orthogonal to $\mathbf{e}_{\mathbf{l}}$ evolves like the center of mass motion in the planar MMC case.

Theorem 1. Let $\mathbf{z}_{\mathbf{x y}} \triangleq\left[\begin{array}{l}z_{x} \\ z_{y}\end{array}\right] \in \mathbb{R}^{2}$ be the projection of $\mathbf{z}$ on the plane orthogonal to $\mathbf{e}_{\mathbf{l}}$, with components $z_{x} \triangleq \mathbf{z} \cdot \mathbf{e}_{\mathbf{x}}$, $z_{y} \triangleq \mathbf{z} \cdot \mathbf{e}_{\mathbf{y}}$. Then $\mathbf{z}_{\mathbf{x y}}$ satisfies (cf. (6)):

$$
\ddot{\mathbf{z}}_{\mathbf{x y}}(t)=\frac{\mu l(0)}{\rho(0) \lambda(0)} \lambda(t) \dot{\mathbf{z}}_{\mathbf{x y}}^{\perp}(t),
$$

where $\lambda=\mathbf{r} \cdot \dot{\mathbf{r}}^{\perp} / \rho \leq 0$.

Proof. The first two components of (20) give $\dot{\mathbf{z}}_{\mathbf{x y}}(t)$. Hence by further differentiating with respect to time:

$$
\ddot{\mathbf{z}}_{\mathbf{x y}}(t)=\dot{\alpha}(t)\left[\begin{array}{c}
\left|\mathbf{e}_{\mathbf{l}} \times \mathbf{k}(0)\right| \cos (-\alpha(t)) \\
-\left|\mathbf{e}_{\mathbf{l}} \times \mathbf{k}(0)\right| \sin (-\alpha(t))
\end{array}\right]=\dot{\alpha}(t) \dot{\mathbf{z}}_{\mathbf{x y}}^{\perp}(t) .
$$

Finally, from (7), (15) and the definition of $\alpha(t)$ :

$\dot{\alpha}(t)=\mu l(t) / \rho(t)=\mu l(0) e^{\mu(\rho(t)-\rho(0))} / \rho(t)=\frac{\mu l(0)}{\rho(0) \lambda(0)} \lambda(t)$.

Here $\lambda$ is computed as in the planar case (4): $\lambda=\mathbf{r} \cdot \dot{\mathbf{r}}^{\perp} / \rho$, where $\dot{\mathbf{r}}^{\perp}=\mathbf{e}_{\mathbf{l}} \times \dot{\mathbf{r}}$ is the (counterclockwise) rotation of $\dot{\mathbf{r}}$ on the plane orthogonal to $\mathbf{e}_{\mathbf{l}}$ (on which both $\mathbf{r}$ and $\dot{\mathbf{r}}$ are constrained). It is easy to verify that $\lambda \leq 0$; in particular $\lambda(0) \leq 0$ and the multiplicative constant in (21) has the same sign as that in (6).

From the results on planar MMC recalled in section 2, the projection of the center of mass motion on the plane orthogonal to $\mathbf{e}_{\mathbf{l}}$ is generically aperiodic and fills in time annular regions on such plane with radii proportional to the relative distance extrema $\rho_{\min }$ and $\rho_{\max }$.

The center of mass motion is therefore the vector sum of a linear motion in the direction of $\mathbf{e}_{\mathbf{l}}$ (dependent on the initial conditions) and a planar MMC motion on a plane orthogonal to $\mathbf{e}_{\mathbf{l}}$. The trajectory is a "modulated" helix, as below.

Theorem 2. The curvature and torsion profiles of the center of mass trajectory in MMC-3D (well-defined provided that $\mathbf{k}(0)$ is not parallel to $\mathbf{e}_{\mathbf{l}}$ ) are given by:

$$
\begin{aligned}
& \kappa(t)=\frac{\beta(t)}{\theta^{2}}\left|\mathbf{k}(0) \times \mathbf{e}_{\mathbf{l}}\right| \\
& \tau(t)=\frac{\beta(t)}{\theta^{2}}\left(\mathbf{k}(0) \cdot \mathbf{e}_{\mathbf{l}}\right),
\end{aligned}
$$

and are identical to those of a "modulated" circular helix, with periodic angular frequency $\beta(t) \triangleq \dot{\alpha}(t)=\mu l(t) / \rho(t)$.

Proof. Recall that, given a thrice-differentiable curve $t \mapsto$ $\mathbf{z}(t) \in \mathbb{R}^{3}$ with $|\dot{\mathbf{z}}(t)|=\theta$ (constant), the curvature and torsion profiles are defined as respectively $\kappa(t) \triangleq|\dot{T}(t)| / \theta$ and $\tau(t) \triangleq(\dot{N}(t) \cdot B(t)) / \theta$. Here $T(t) \triangleq \dot{\mathbf{z}}(t) / \theta, N(t) \triangleq$ $\dot{T}(t) /|\dot{T}(t)|$ and $B(t) \triangleq T(t) \times N(t)$ are the tangent, normal and binormal to the curve, which form its Frenet-Serret frame (see for example Bishop [1975]).

It is easy to verify that for a circular helix having angular frequency $\omega$, radius $|a| / \omega$, pitch $2 \pi b / \omega$ and constant speed $\sqrt{a^{2}+b^{2}}$ (e.g. of the form $\mathbf{z}(t)=(a / \omega) \cos (\omega t) \mathbf{e}_{\mathbf{x}}+$ $(a / \omega) \sin (\omega t) \mathbf{e}_{\mathbf{y}}+b t \mathbf{e}_{\mathbf{l}}$ if the helix axes is $\left.\mathbf{e}_{\mathbf{l}}\right)$, the curvature and torsion profiles are constant and given by:

$\kappa(t)=\frac{|a| \omega}{a^{2}+b^{2}} \forall t, \tau(t)=\frac{b \omega}{a^{2}+b^{2}} \forall t$.

Before computing the curvature and torsion for the center of mass trajectory, we remark the following consequences of the fact that $\dot{\mathbf{k}}(t)=-\beta(t)\left(\mathbf{k}(t) \times \mathbf{e}_{\mathbf{l}}\right)$ :

$$
\begin{aligned}
\frac{d}{d t}\left|\mathbf{k}(t) \times \mathbf{e}_{\mathbf{l}}\right| & =\frac{\left(\mathbf{k}(t) \times \mathbf{e}_{\mathbf{l}}\right) \cdot\left(\dot{\mathbf{k}}(t) \times \mathbf{e}_{\mathbf{l}}\right)}{\left|\mathbf{k}(t) \times \mathbf{e}_{\mathbf{l}}\right|}=0 \quad \forall t \\
\frac{d}{d t}\left(\mathbf{k}(t) \cdot \mathbf{e}_{\mathbf{l}}\right) & =\dot{\mathbf{k}}(t) \cdot \mathbf{e}_{\mathbf{l}}=0 \quad \forall t .
\end{aligned}
$$

Hence, $\forall t>0,\left|\mathbf{k}(t) \times \mathbf{e}_{\mathbf{l}}\right|=\left|\mathbf{k}(0) \times \mathbf{e}_{\mathbf{l}}\right|$ (which we assume nonzero) and $\mathbf{k}(t) \cdot \mathbf{e}_{\mathbf{l}}=\mathbf{k}(0) \cdot \mathbf{e}_{\mathbf{l}}$ are constant. Exploiting these facts, we can compute the relevant quantities for the center of mass trajectory as follows:

$$
\begin{aligned}
& T(t)=\frac{\mathbf{k}(\mathbf{t})}{\theta}, \quad \dot{T}(t)=\frac{\dot{\mathbf{k}}(t)}{\theta}=\frac{-\beta(t)\left(\mathbf{k}(t) \times \mathbf{e}_{\mathbf{l}}\right)}{\theta}, \\
& N(t)=\frac{\dot{T}(t)}{|\dot{T}(t)|}=\frac{-\mathbf{k}(t) \times \mathbf{e}_{\mathbf{l}}}{\left|\mathbf{k}(0) \times \mathbf{e}_{\mathbf{l}}\right|}, \\
& \dot{N}(t)=\frac{-\mathbf{k}(t) \times \mathbf{e}_{\mathbf{l}}}{\left|\mathbf{k}(0) \times \mathbf{e}_{\mathbf{l}}\right|}=\beta(t) \frac{\mathbf{e}_{\mathbf{l}}\left(\mathbf{k}(0) \cdot \mathbf{e}_{\mathbf{l}}\right)-\mathbf{k}(t)}{\left|\mathbf{k}(0) \times \mathbf{e}_{\mathbf{l}}\right|}, \\
& B(t)=T(t) \times N(t)=\frac{-\mathbf{k}(t)\left(\mathbf{k}(0) \cdot \mathbf{e}_{\mathbf{l}}\right)+\mathbf{e}_{\mathbf{l}} \theta^{2}}{\theta\left|\mathbf{k}(0) \times \mathbf{e}_{\mathbf{l}}\right|} .
\end{aligned}
$$

So the curvature and torsion of the center of mass trajectory are indeed given by:

$$
\begin{aligned}
& \kappa(t)=\frac{|\dot{T}(t)|}{\theta}=\frac{\beta(t)}{\theta^{2}}\left|\mathbf{k}(0) \times \mathbf{e}_{\mathbf{l}}\right|, \\
& \tau(t)=\frac{\dot{N}(t) \cdot B(t)}{\theta} \frac{\beta(t)}{\theta^{2}}\left(\mathbf{k}(0) \cdot \mathbf{e}_{\mathbf{l}}\right) .
\end{aligned}
$$

If we compare with the curvature and torsion of a standard circular helix, we see that there is an exact correspondence between the center of mass trajectory and a "modulated" helix with periodic angular frequency $\beta(t)$, radius $\mid \mathbf{k}(0) \times$ $\mathbf{e}_{\mathbf{l}} \mid / \beta(t)$, pitch $2 \pi\left(\mathbf{k}(0) \cdot \mathbf{e}_{\mathbf{l}}\right) / \beta(t)$ and constant speed $\theta$. 
It is indeed the "modulation" in the angular frequency (driven by the changes in relative distance between the agents) that makes the trajectory more complicated (and interesting) than a standard circular helix.

\subsection{Reconstruction of individual trajectories}

Since $\mathbf{r}_{1}=(\mathbf{z}+\mathbf{r}) / 2$ and $\mathbf{r}_{\mathbf{2}}=(\mathbf{z}-\mathbf{r}) / 2$, the individual dynamics are given by:

$$
\begin{aligned}
& \ddot{\mathbf{r}}_{\mathbf{1}}=\frac{1}{2}(\ddot{\mathbf{z}}+\ddot{\mathbf{r}})=\frac{\mu}{\rho}\left(\mathbf{l} \times \dot{\mathbf{r}}_{\mathbf{1}}\right)=\frac{\mu l}{\rho} \hat{\mathbf{e}}_{\mathbf{1}} \dot{\mathbf{r}}_{\mathbf{1}} \\
& \ddot{\mathbf{r}}_{\mathbf{2}}=\frac{1}{2}(\ddot{\mathbf{z}}-\ddot{\mathbf{r}})=\frac{\mu}{\rho}\left(\mathbf{l} \times \dot{\mathbf{r}}_{\mathbf{2}}\right)=\frac{\mu l}{\rho} \hat{\mathbf{e}}_{\mathbf{1}} \dot{\mathbf{r}}_{\mathbf{2}},
\end{aligned}
$$

which means that $\mathbf{r}_{1}$ and $\mathbf{r}_{2}$ satisfy:

$$
\dot{\mathbf{r}}_{\mathbf{i}}(t)=\operatorname{Rot}\left(\mathbf{e}_{\mathbf{l}}, \alpha(t)\right) \dot{\mathbf{r}}_{\mathbf{i}}(0), \quad i=1,2,
$$

with $\alpha(t) \triangleq \mu \int_{0}^{t} \frac{l(s)}{\rho(s)} d s$. The motion of the $i$-th agent $(i=1,2)$ is rectilinear if $\dot{\mathbf{r}}_{\mathbf{i}}(0)$ is parallel to $\mathbf{e}_{\mathbf{l}}$, or if $\mathbf{e}_{\mathbf{l}}=\mathbf{0}$. In the other cases, it can be decomposed with respect to the orthonormal frame $\left(\mathbf{e}_{\mathbf{x}}, \mathbf{e}_{\mathbf{y}}, \mathbf{e}_{\mathbf{l}}\right)$ as done for the center of mass:

$$
\dot{\mathbf{r}}_{\mathbf{i}}(t)=\left[\begin{array}{c}
\dot{r}_{i x}(t) \\
\dot{r}_{i y}(t) \\
\dot{r}_{i l}(t)
\end{array}\right]=\left[\begin{array}{c}
-\left|\mathbf{e}_{\mathbf{l}} \times \dot{\mathbf{r}}_{\mathbf{i}}(0)\right| \sin (-\alpha(t)) \\
-\left|\mathbf{e}_{\mathbf{l}} \times \dot{\mathbf{r}}_{\mathbf{i}}(0)\right| \cos (-\alpha(t)) \\
\dot{\mathbf{r}}_{\mathbf{i}}(0) \cdot \mathbf{e}_{\mathbf{l}}
\end{array}\right] .
$$

Therefore each of the agents travels at constant speed $\nu_{i}$ along a "modulated" circular helix of the type described in the previous subsection, with axis $\mathbf{e}_{\mathbf{l}}$ and curvature and torsion profiles:

$$
\begin{aligned}
\kappa_{i}(t) & =\frac{\beta(t)}{\nu_{i}^{2}}\left|\dot{\mathbf{r}}_{\mathbf{i}}(0) \times \mathbf{e}_{\mathbf{l}}\right| \\
\tau_{i}(t) & =\frac{\beta(t)}{\nu_{i}^{2}}\left(\dot{\mathbf{r}}_{\mathbf{i}}(0) \cdot \mathbf{e}_{\mathbf{l}}\right) .
\end{aligned}
$$

Figure 1 shows a typical set of trajectories obtained for two agents engaged in Mutual Motion Camouflage in $\mathbb{R}^{3}$. Notice that the agent speeds are different in this example.

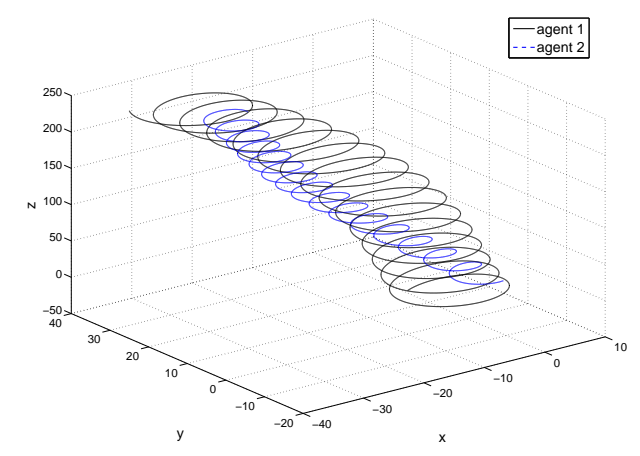

Fig. 1. Representative trajectories obtained with MMC-3D

\subsection{Special cases}

Among the trajectories that can be obtained with MMC$3 \mathrm{D}$, we highlight some interesting special cases and suitable choices of initial conditions to achieve them.

(i) Rectilinear motion of both agents

Both agents have constant velocity, and hence move in straight lines, if $\mathbf{e}_{\mathbf{l}}=\mathbf{0} \Rightarrow \mathbf{r}(0) \times \dot{\mathbf{r}}(0)=\mathbf{0}$. Since we exclude collision configurations (otherwise the MC control laws are not well-defined), this can happen only if either $\dot{\mathbf{r}}(0)=\nu_{1} \mathbf{x}_{1}-\nu_{2} \mathbf{x}_{2}=\mathbf{0}$ or if $\dot{\mathbf{r}}(0)$ is parallel to $\mathbf{r}(0)$. The first case is possible only if the agent speeds are equal $\left(\nu_{1}=\nu_{2}\right)$, and their initial directions of motion are identical; in this case the agents move along parallel lines. The second case corresponds to $\Gamma(\mathbf{r}(0), \dot{\mathbf{r}}(0))= \pm 1$. Just as in planar MMC, the sets $\Gamma(\mathbf{r}, \dot{\mathbf{r}})= \pm 1$ are (non-attracting) invariant manifolds, and therefore the agents remain in a state of motion camouflage with the distance reducing till collision (if $\Gamma=-1$ ) or increasing (if $\Gamma=1$ ).

(ii) Rectilinear motion of one of the agents

The $i$-th agent moves along a straight line if $\dot{\mathbf{r}}_{\mathbf{i}}(0)=\nu_{i} \mathbf{e}_{\mathbf{l}}$. By the definition of $\mathbf{e}_{\mathbf{l}}$, this requires $\dot{\mathbf{r}}_{\mathbf{i}}(0)$ to be orthogonal to $\mathbf{r}(0)$. For given initial relative position $\mathbf{r}(0)$, and desired initial velocity (and hence direction of the straight line trajectory) for one of the agents (say $\dot{\mathbf{r}}_{\mathbf{1}}(0)=\nu_{1} \mathbf{x}_{\mathbf{1}}(0) \perp$ $\mathbf{r}(0)$ ), the only choice of velocity for the other agent that guarantees that $\mathbf{e}_{\mathbf{1}}=\dot{\mathbf{r}}_{\mathbf{1}}(0) / \nu_{1}$ is: $\dot{\mathbf{r}}_{\mathbf{2}}(0)=\nu_{2} \mathbf{x}_{\mathbf{2}}(0)=$ $\nu_{1} \mathbf{x}_{\mathbf{1}}(0)+\left(\mathbf{r}(0) \times \mathbf{x}_{\mathbf{1}}(0)\right)$.

(iii) Rectilinear motion of the center of mass

The center of mass moves along a straight line if $\mathbf{k}(0)$ is parallel to $\mathbf{e}_{\mathbf{l}}$. Let $\mathbf{r}(0)$ be the initial relative position between the agents, and $\mathbf{e}_{\mathbf{l}}$ a vector orthogonal to $\mathbf{r}(0)$ (the desired direction for the center of mass motion). For $\mathbf{k}(0)$ to be parallel to $\mathbf{e}_{\mathbf{l}}$, the initial relative velocity $\mathbf{g}(0)$ must satisfy the condition $\mathbf{g}(0) /|\mathbf{g}(0)|=\mathbf{r}(0) \times \mathbf{e}_{\mathbf{l}} / \mid \mathbf{r}(0) \times$ $\mathbf{e}_{\mathbf{l}} \mid$. Since $\mathbf{k}(0)$ must be parallel to $\mathbf{l}(0)=\mathbf{r}(0) \times \mathbf{g}(0)$, it must also be orthogonal to $\mathbf{g}(0)$, and thus $2 \nu_{1}=\mid \mathbf{k}(0)+$ $\mathbf{g}(0)|=| \mathbf{k}(0)-\mathbf{g}(0) \mid=2 \nu_{2}$. Hence the two agent speeds must be equal: $\nu_{1}=\nu_{2} \triangleq \nu$. The magnitudes $\delta=|\mathbf{g}(0)|$ and $\theta=|\mathbf{k}(0)|$ must be related to the common speed by the relation $\sqrt{\delta^{2}+\theta^{2}}=2 \nu$ (if $\theta=0$ we have the planar mutual motion camouflage described in (v)). Provided that these conditions are satisfied, the following initial directions of motion for the agents guarantee that the center of mass will move in the direction $\mathbf{e}_{\mathbf{l}}$ at speed $\theta$ :

$\mathbf{x}_{\mathbf{1}}(0)=\frac{\mathbf{k}(0)+\mathbf{g}(0)}{2 \nu}=\frac{\delta}{2 \nu} \frac{\mathbf{r}(0) \times \mathbf{e}_{1}}{\left|\mathbf{r}(0) \times \mathbf{e}_{\mathbf{1}}\right|}+\frac{\theta}{2 \nu} \mathbf{e}_{\mathbf{l}}$,
$\mathbf{x}_{\mathbf{2}}(0)=\frac{\mathbf{k}(0)-\mathbf{g}(0)}{2 \nu}=\frac{\theta}{2 \nu} \mathbf{e}_{\mathbf{l}}-\frac{\delta}{2 \nu} \frac{\mathbf{r}(0) \times \mathbf{e}_{\mathbf{1}}}{\left|\mathbf{r}(0) \times \mathbf{e}_{\mathbf{1}}\right|}$.

(iv) Motion of both agents along a double helix

The trajectories of the agents (and of the center of mass) are true circular helices in the special case that the relative distance $\rho(t)$ remains constant in time. In that case in fact $\beta(t)=\dot{\alpha}(t)$ is constant in the curvature and torsion profiles (28)-(29). This occurs if the initial conditions fall on the equilibrium point $\rho(0)=1 / \mu, \dot{\rho}(0)=0$ of the relative distance dynamics. Notice that $\dot{\rho}(0)=\gamma(0)=(\mathbf{g}(0)$. $\mathbf{r}(0)) /|\mathbf{r}(0)|=0$ requires $\mathbf{g}(0)$ to be orthogonal to $\mathbf{r}(0)$ (and of course to $\left.\mathbf{e}_{\mathbf{l}}\right)$. Hence this special motion is achieved if initially the positions of the agents have distance $1 / \mu$ and their velocities satisfy:

$\frac{\mathbf{g}(0)}{|\mathbf{g}(0)|}=\frac{\nu_{1} \mathbf{x}_{\mathbf{1}}(0)-\nu_{2} \mathbf{x}_{\mathbf{2}}(0)}{\left|\nu_{1} \mathbf{x}_{\mathbf{1}}(0)-\nu_{2} \mathbf{x}_{\mathbf{2}}(0)\right|}=\frac{\mathbf{r}(0) \times \mathbf{e}_{\mathbf{1}}}{\rho(0)}=\mu\left(\mathbf{r}(0) \times \mathbf{e}_{\mathbf{l}}\right)$.

When this is the case: $\beta(t)=\dot{\alpha}(t)=\mu l(t) / \rho(t)=$ $\mu^{2}|\mathbf{l}(0)|=\mu^{2}|\mathbf{r}(0) \times \mathbf{g}(0)|=\mu^{2} \delta \rho(0)=\mu \delta$, where $\delta=$ $|\mathbf{g}(0)|=\left|\nu_{1} \mathbf{x}_{\mathbf{1}}(0)-\nu_{2} \mathbf{x}_{\mathbf{2}}(0)\right|$. The curvature and torsion for each agent, given by the constants $\kappa_{i}(t)=\mu \delta\left|\dot{\mathbf{r}}_{\mathbf{i}}(0) \times \mathbf{e}_{\mathbf{l}}\right| / \nu_{i}^{2}$ and $\tau_{i}(t)=\mu \delta\left(\dot{\mathbf{r}}_{\mathbf{i}}(0) \cdot \mathbf{e}_{\mathbf{l}}\right) / \nu_{i}^{2}$, correspond to circular helices with angular frequency $\mu \delta$, pitch $2 \pi\left(\dot{\mathbf{r}}_{\mathbf{i}}(0) \cdot \mathbf{e}_{\mathbf{l}}\right) /(\mu \delta)$ and radius $\left|\dot{\mathbf{r}}_{\mathbf{i}}(0) \times \mathbf{e}_{\mathbf{l}}\right| /(\mu \delta)$, covered at speed $\nu_{i}$. 


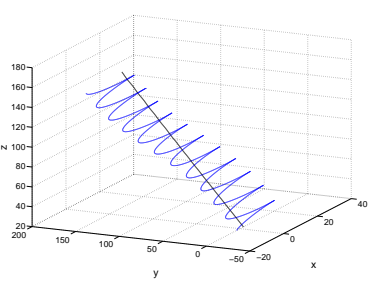

(a)

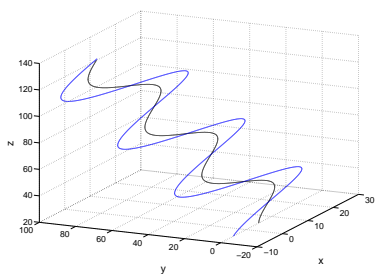

(c)

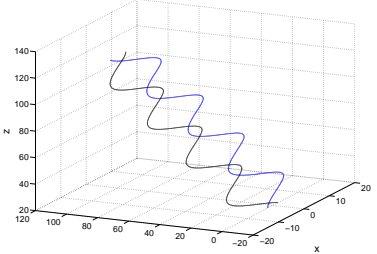

(b)

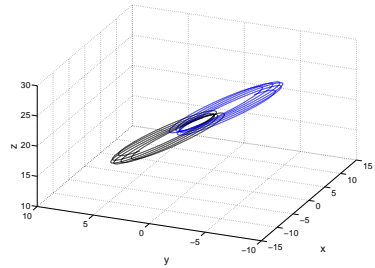

(d)
Fig. 2. Examples of special trajectories obtained with MMC-3D: (a) rectilinear motion of one agent; (b) rectilinear motion of the center of mass; (c) double helix; (d) planar MMC.

(v) Planar mutual motion camouflage

The last special case is the one in which $\mathbf{k}(0)=\mathbf{0}$. Then $\mathbf{k}(t)=\mathbf{0} \forall t \geq 0$ and thus $\dot{\mathbf{r}}_{\mathbf{1}}(t)=\dot{\mathbf{r}}(t) / 2, \dot{\mathbf{r}}_{\mathbf{2}}(t)=-\dot{\mathbf{r}}(t) / 2$. From the results of section 4.1, each agent moves on the fixed plane orthogonal to $\mathbf{e}_{\mathbf{l}}$ (the direction of $\mathbf{l}(0)$ ), and the two agents perform a planar mutual motion camouflage on that plane. For the MMC-3D system to fall into this special case, with motion constrained on the plane orthogonal to a prescribed $\mathbf{e}_{\mathbf{l}}$, the initial positions and velocities of the agents must satisfy the constraints $\nu_{1} \mathbf{x}_{\mathbf{1}}(0)=-\nu_{2} \mathbf{x}_{\mathbf{2}}(0)$ and $\frac{\left(\mathbf{r}_{1}(0)-\mathbf{r}_{2}(0)\right) \times \nu_{1} \mathbf{x}_{1}(0)}{\left|\left(\mathbf{r}_{1}(0)-\mathbf{r}_{2}(0)\right) \times \nu_{1} \mathbf{x}_{1}(0)\right|}=\mathbf{e}_{\mathbf{l}}$. The first constraint can be satisfied only if the speeds of the agents are identical.

Figure 2 shows examples of the special trajectories described above (except the simple case of rectilinear motion of both agents).

\section{MMC-3D FOR SURVEILLANCE}

The trajectories of the agents in MMC-3D are the vector sum of a constant-speed translation in the direction $\mathbf{e}_{\mathbf{l}}$, and a planar mutual motion camouflage on the plane orthogonal to $\mathbf{e}_{\mathbf{l}}$. Since the planar MMC trajectories have useful region-filling characteristics (they provide dense coverage of certain annular regions), the MMC-3D model can be made potentially useful for surveillance applications (aerial coverage of regions of interest) by adding control authority on the motion in the direction of $\mathbf{e}_{\mathbf{l}}$. This can be accomplished with appropriate initial conditions and the following modifications of the MMC-3D control laws.

Theorem 3. Let the conditions $\nu_{1}=\nu_{2} \triangleq \nu$ and $\mathbf{k}(0)$ parallel to $\mathbf{l}(0) \neq \mathbf{0}$ be satisfied (cf. section 4.4-(iii)), and let the curvature control laws for the two agents be given by:

$$
\begin{aligned}
u_{i} & =u_{i, M M C}+u_{i}^{\prime}, \quad v_{i}=v_{i, M M C}+v_{i}^{\prime}, \\
u_{i}^{\prime} & =-\frac{\mu}{\nu_{i}}\left[\mathbf{y}_{\mathbf{i}} \cdot\left(\frac{a}{\nu_{i}} \frac{\mathbf{k}}{|\dot{\mathbf{r}}|^{2}}+\frac{b}{\nu_{i}|\dot{\mathbf{r}}|^{2}}\left(\mathbf{z} \cdot \mathbf{e}_{\mathbf{l}}-\tilde{z}_{l}\right) \mathbf{e}_{\mathbf{l}}\right)\right] \\
v_{i}^{\prime} & =-\frac{\mu}{\nu_{i}}\left[\mathbf{z}_{\mathbf{i}} \cdot\left(\frac{a}{\nu_{i}} \frac{\mathbf{k}}{|\dot{\mathbf{r}}|^{2}}+\frac{b}{\nu_{i}|\dot{\mathbf{r}}|^{2}}\left(\mathbf{z} \cdot \mathbf{e}_{\mathbf{l}}-\tilde{z}_{l}\right) \mathbf{e}_{\mathbf{l}}\right)\right],
\end{aligned}
$$

where $u_{i, M M C}, v_{i, M M C}$ are the curvature controls of MMC (given by (11)), $a>0, b>0$ are suitable constant gains, $\mathbf{e}_{\mathbf{l}}=\mathbf{l} /|\mathbf{l}|$ is constant, and $\tilde{z}_{l}$ is the desired position of the scaled center of mass $\mathbf{z}(t)$ in the direction of $\mathbf{e}_{\mathbf{l}}$. Then $\mathbf{z}(t)$ moves along a straight line and converges asymptotically from its initial position $\mathbf{z}(0)$ to the desired position $\mathbf{z}(0)+$ $\left(\tilde{z}_{l}-\left(\mathbf{z}(0) \cdot \mathbf{e}_{\mathbf{l}}\right)\right) \mathbf{e}_{\mathbf{l}}$, while the relative motion between the agents remains constrained on the plane orthogonal to $\mathbf{e}_{\mathbf{l}}$ and converges to a planar MMC with $\delta=2 \nu$. As a result, the agent trajectories asymptotically fill annular regions centered at $\left(\mathbf{z}(0)+\left(\tilde{z}_{l}-\left(\mathbf{z}(0) \cdot \mathbf{e}_{\mathbf{l}}\right)\right) \mathbf{e}_{\mathbf{l}}\right) / 2$ and lying on a plane orthogonal to $\mathbf{e}_{\mathbf{l}}$.

Proof. The closed-loop equations obtained substituting (30)-(32) in the agent dynamics (9) are (here $\mathbf{g}=\dot{\mathbf{r}}, \mathbf{k}=\dot{\mathbf{z}}$ as before, and $\left.z_{l} \triangleq \mathbf{z} \cdot \mathbf{e}_{\mathbf{l}}\right)$ :

$$
\begin{aligned}
& \dot{\mathbf{g}}=-\frac{\mu}{\rho}(\mathbf{g} \times \mathbf{l})+\frac{\mu a}{|\mathbf{g}|^{2}}\left(2-\frac{|\mathbf{g}|^{2}}{2 \nu^{2}}\right) \mathbf{g}+\frac{\mu b\left(z_{l}-\tilde{z}_{l}\right)}{2 \nu^{2}|\mathbf{g}|^{2}}\left(\mathbf{k} \cdot \mathbf{e}_{\mathbf{l}}\right) \mathbf{g} \\
& \dot{\mathbf{k}}=-\frac{\mu}{\rho}(\mathbf{k} \times \mathbf{l})-\frac{\mu a}{2 \nu^{2}} \mathbf{k}-\frac{\mu b}{|\mathbf{g}|^{2}}\left(z_{l}-\tilde{z}_{l}\right)\left(2 \mathbf{e}_{\mathbf{l}}-\frac{\mathbf{k} \cdot \mathbf{e}_{\mathbf{l}}}{2 \nu^{2}} \mathbf{k}\right),
\end{aligned}
$$

Observe that $\mathbf{e}_{\mathbf{l}}$, the direction of the relative angular momentum $\mathbf{l}$, is constant in time, since:

$$
\mathbf{i}=\left[\mu \gamma+\frac{\mu a}{|\mathbf{g}|^{2}}\left(2-\frac{|\mathbf{g}|^{2}}{2 \nu^{2}}\right)+\frac{\mu b\left(z_{l}-\tilde{z}_{l}\right)}{2 \nu^{2}|\mathbf{g}|^{2}}\left(\mathbf{k} \cdot \mathbf{e}_{\mathbf{l}}\right)\right] \mathbf{l}
$$

Let us first consider the center of mass dynamics (34). If $\mathbf{k}(0)$ is parallel to $\mathbf{e}_{\mathbf{l}}$, then so is $\mathbf{k}(t) \forall t$ (since in this case $\dot{\mathbf{k}}$ is parallel to $\mathbf{k}$ ). Hence $\mathbf{k}=\dot{\mathbf{z}}=\dot{z}_{l} \mathbf{e}_{\mathbf{l}}$ and $\dot{\mathbf{k}}=\ddot{\mathbf{z}}=$ $\ddot{z}_{l} \mathbf{e}_{\mathbf{l}}$. Therefore (34) can be reduced to a scalar secondorder differential equation in $\Delta z_{l} \triangleq z_{l}-\tilde{z}_{l}$, obtained by projection in the direction $\mathbf{e}_{\mathbf{l}}$ :

$$
\Delta \ddot{z}_{l}+\frac{\mu a}{2 \nu^{2}} \Delta \dot{z}_{l}+\frac{\mu b}{2 \nu^{2}} \Delta z_{l}=0 .
$$

Here we have used the fact that $2-\left(\mathbf{k} \cdot \mathbf{e}_{\mathbf{l}}\right)^{2} /\left(2 \nu^{2}\right)=2-$ $|\mathbf{k}|^{2} /\left(2 \nu^{2}\right)=|\mathbf{g}|^{2} /\left(2 \nu^{2}\right)$ when $\mathbf{k}$ is in the direction $\mathbf{e}_{\mathbf{l}}$ and $\nu_{1}=\nu_{2}=\nu\left(\right.$ since $|\mathbf{k}+\mathbf{g}|=|\mathbf{k}-\mathbf{g}|=\sqrt{|\mathbf{k}|^{2}+|\mathbf{g}|^{2}}=2 \nu$ in this case). The linear constant-coefficient differential equation (36) has solutions that asymptotically converge to $\Delta z_{l}=0$, for all initial conditions $\Delta z_{l}(0), \Delta \dot{z}_{l}(0)$.

The control laws (30)-(32) are well-defined because $|\mathbf{g}(t)|=$ $|\dot{\mathbf{r}}(t)| \neq 0 \forall t$ when $a, b$ are chosen appropriately. To show that this is the case, consider the dynamics of the (nonpositive) variable $\Delta \delta \triangleq|\mathbf{g}|^{2}-4 \nu^{2}=-|\mathbf{k}|^{2}$ :

$$
\Delta \dot{\delta}=2(\mathbf{g} \cdot \dot{\mathbf{g}})=-\frac{\mu a}{\nu^{2}} \Delta \delta+\frac{\mu b}{\nu^{2}} \Delta z_{l} \Delta \dot{z}_{l}
$$

We can see this equation as a linear system driven (or perturbed) by a time-varying input $\Delta z_{l} \Delta \dot{z}_{l}$. The evolution of $\Delta z_{l}(t)$ and $\Delta \dot{z}_{l}(t)$ can be computed analytically, and so does the solution to (37). Assume for example that the constants $a>0, b>0$ are chosen in such a way that the characteristic polynomial of (36) has two real and distinct roots $\lambda_{2}<\lambda_{1}<0$. Notice that $\lambda_{1,2}=$ 
$\left(-\mu a \pm \sqrt{\mu^{2} a^{2}-8 \mu b \nu^{2}}\right) /\left(4 \nu^{2}\right)$ hence this requires that $b<$ $\left(\mu a^{2}\right) /\left(8 \nu^{2}\right)$. Then the solution of (36) for initial conditions $\Delta z_{l}(0), \Delta \dot{z}_{l}(0)$ is given by: $\Delta z_{l}(t)=c_{1} e^{\lambda_{1} t}+c_{2} e^{\lambda_{2} t}$, where $c_{1}=\left(\Delta z_{l}(0) \lambda_{2}-\Delta \dot{z}_{l}(0)\right) /\left(\lambda_{2}-\lambda_{1}\right), c_{2}=\left(\Delta \dot{z}_{l}(0)-\right.$ $\left.\Delta z_{l}(0) \lambda_{1}\right) /\left(\lambda_{2}-\lambda_{1}\right)$. The evolution of $\Delta \delta(t)$ starting from an initial value $\Delta \delta(0)>-4 \nu^{2}$ (since $\mathbf{l}(0) \neq \mathbf{0}$, it must be $\left.|\mathbf{g}(0)|^{2}>0\right)$ is therefore:

$$
\begin{aligned}
\Delta \delta(t)= & e^{\frac{-\mu a}{\nu^{2}} t} \Delta \delta(0)+\frac{\mu b}{\nu^{2}} \int_{0}^{t} e^{\frac{-\mu a}{\nu^{2}}(t-\tau)} \Delta z_{l}(\tau) \Delta \dot{z}_{l}(\tau) d \tau \\
= & e^{\frac{-\mu a}{\nu^{2}} t}\left(\Delta \delta(0)-c_{3}-c_{4}-c_{5}\right) \\
& +c_{3} e^{2 \lambda_{1} t}+c_{4} e^{2 \lambda_{2} t}+c_{5} e^{\left(\lambda_{1}+\lambda_{2}\right) t}
\end{aligned}
$$

where $c_{3}=\mu b \lambda_{1} c_{1}^{2} /\left(2 \nu^{2} \lambda_{1}+\mu a\right)<0, c_{4}=\mu b \lambda_{2} c_{2}^{2} /\left(2 \nu^{2} \lambda_{2}+\right.$ $\mu a)<0$ and $c_{5}=\mu b\left(\lambda_{1}+\lambda_{2}\right) c_{1} c_{2} /\left(\nu^{2}\left(\lambda_{1}+\lambda_{2}\right)+\mu a\right)=$ $-\mu b c_{1} c_{2} / 2$. By $(38), \Delta \delta(t) \geq-\left|\Delta \delta(0)-c_{3}-c_{4}-c_{5}\right|-$ $\left|c_{3}\right|-\left|c_{4}\right|-\left|c_{5}\right| \forall t$, and since $c_{3}(a, b) \stackrel{b \rightarrow 0}{\longrightarrow} 0, c_{4}(a, b) \stackrel{b \rightarrow 0}{\longrightarrow} 0$, $c_{5}(a, b) \stackrel{b \rightarrow 0}{\longrightarrow} 0$ (and $\left.\Delta \delta(0)>-4 \nu^{2}\right)$, it is always possible to choose $b$ sufficiently small to guarantee that $\Delta \delta(t)>$ $-4 \nu^{2} \forall t$, and hence $|\mathbf{g}(t)|^{2}>0 \forall t$. This proves that the control laws are well-defined (at least for suitable choices of the gains $a, b)$. Furthermore, $(38)$ shows that $\Delta \delta(t)$ goes to zero asymptotically, and therefore the relative speed converges to the value $\delta=2 \nu$. Finally, let us consider the relative motion dynamics (33). As $\Delta z_{l} \rightarrow 0$ and $|\mathbf{g}|^{2} \rightarrow 4 \nu^{2}$ asymptotically, these dynamics converge to those of the standard MMC-3D case (16) (with constant relative speed $\delta=2 \nu)$.

Another modification of MMC-3D that can be useful for surveillance applications is a three-dimensional version of (8); the objective of this modification is to stabilize the energy of the (periodic) relative distance dynamics to a desired value, to indirectly control the minimum and maximum distances between the agents.

Theorem 4. Let the curvature control laws for the two agents be given by:

$$
\begin{aligned}
u_{i} & =u_{i, M M C}+u_{i}^{\prime \prime}, \quad v_{i}=v_{i, M M C}+v_{i}^{\prime \prime}, \\
u_{i}^{\prime \prime} & =\left[\frac{k_{d}}{\nu_{i}}\left(\frac{\mathbf{r} \cdot \dot{\mathbf{r}}}{|\mathbf{r}|}\right)\left(E-E_{d}\right)\right]\left[\mathbf{z}_{\mathbf{i}} \cdot\left(\dot{\mathbf{r}} \times \frac{\mathbf{r}}{|\mathbf{r}|}\right)\right] \\
v_{i}^{\prime \prime} & =-\left[\frac{k_{d}}{\nu_{i}}\left(\frac{\mathbf{r} \cdot \dot{\mathbf{r}}}{|\mathbf{r}|}\right)\left(E-E_{d}\right)\right]\left[\mathbf{y}_{\mathbf{i}} \cdot\left(\dot{\mathbf{r}} \times \frac{\mathbf{r}}{|\mathbf{r}|}\right)\right],
\end{aligned}
$$

where $u_{i, M M C}, v_{i, M M C}$ are the MMC curvature controls given by (11), $k_{d}>0$ is a constant gain and $0<E_{d} \leq$ $\delta^{2} e^{-2} / \mu^{2}$. Then the energy function $E=E(\rho, \dot{\rho})=\rho^{2}\left(\delta^{2}-\right.$ $\left.\dot{\rho}^{2}\right) e^{-2 \mu \rho}$ converges to the value $E_{d}$, provided that $\dot{\rho}(0) \neq$ $\pm \delta$ and $(\rho(0), \dot{\rho}(0)) \neq(1 / \mu, 0)$.

Proof. The relative motion closed-loop system is:

$$
\ddot{\mathbf{r}}=\left[\mu-k_{d}\left(\frac{\mathbf{r} \cdot \dot{\mathbf{r}}}{|\mathbf{r}|}\right)\left(E-E_{d}\right)\right]\left[\dot{\mathbf{r}} \times\left(\dot{\mathbf{r}} \times \frac{\mathbf{r}}{|\mathbf{r}|}\right)\right] \text {. }
$$

The relative position $\mathbf{r}$ is constrained to the plane orthogonal to $\mathbf{e}_{\mathbf{l}}$, and its dynamics on this plane are identical to those obtained in the two-dimensional model with the control laws (8). The level set $E(\rho, \dot{\rho})=E_{d}$ is an attractor for these dynamics, with region of attraction $\{(\rho, \dot{\rho}): \rho>0,-\delta<\dot{\rho}<\delta,(\rho, \dot{\rho}) \neq(1 / \mu, 0)\}$ (see theorem 2 in Mischiati and Krishnaprasad [2010]). As the energy $E$ converges to the desired value $E_{d},(42)$ converges asymptotically to (12) (and it could also be shown that the center of mass dynamics converge to (13)).
Corollary 1. With the following choices of $\mu$ and $E_{d}$, the control laws (39)-(41) yield (asymptotic) trajectories in which the distance between the agents oscillates between $\hat{\rho}_{\min }$ and $\hat{\rho}_{\max }$ :

$$
\mu=\frac{\ln \left(\hat{\rho}_{\max } / \hat{\rho}_{\min }\right)}{\hat{\rho}_{\max }-\hat{\rho}_{\min }}, \quad E_{d}=\hat{\rho}_{\min }^{2} \delta^{2} e^{-2 \mu \hat{\rho}_{\min }} .
$$

Proof. Immediate from theorem 4 and the fact that the minimum and maximum distances between the agents are the two solutions of equation $\rho^{2} \delta^{2} e^{-2 \mu \rho}=E$.

As an example of surveillance application, we propose the following control strategy that allows two agents (e.g. UAVs) moving at identical and constant speeds $\nu_{1}=\nu_{2}=$ $\nu$ and initially on the ground, to asymptotically cover an annular region of prescribed radii $\hat{\rho}_{\text {min }}$ and $\hat{\rho}_{\max }$ at a fixed prescribed altitude $\tilde{z}_{l}$ :

(i) Choose initial conditions such that $\mathbf{k}(0)$ and $\mathbf{l}(0)$ are both in the vertical direction, compatibly with the hypothesis of theorem 3 (specialized to the case of vertical rectilinear motion of the center of mass).

(ii) Choose $a>0, b>0$ for the "altitude control" component of the control laws, as in theorem 3 .

(iii) Choose $k_{d}>0, \mu>0$ and $0<E_{d} \leq \delta^{2} e^{-2} / \mu^{2}$ for the "energy control" component of the control laws, as in corollary 1 . In this context, the asymptotic value of the relative speed $(\delta=2 \nu)$ should be used.

(iv) Apply a combined control of altitude and energy:

$$
u_{i}=u_{i, M M C}+u_{i}^{\prime}+u_{i}^{\prime \prime}, \quad v_{i}=v_{i, M M C}+v_{i}^{\prime}+v_{i}^{\prime \prime} .
$$

Notice that the closed loop equations due to these control laws are identical to (33)-(34) except that the first terms (in both equations) are scaled by $\mu-k_{d}\left(\frac{\mathbf{r} \cdot \dot{\mathbf{r}}}{|\mathbf{r}|}\right)\left(E-E_{d}\right)$ instead of simply $\mu$. Therefore the center of mass motion (under the special conditions for rectilinear motion) is unaffected by the "energy control", and the relative motion converges asymptotically to (42).

Figure 3 shows a simulation of this control strategy in action.

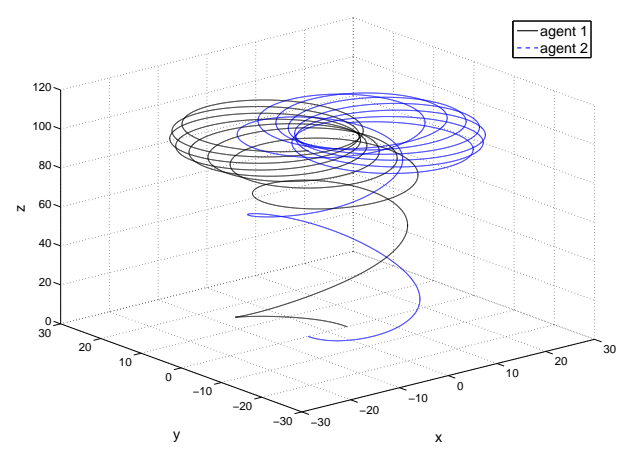

Fig. 3. Asymptotic coverage of an annular region at fixed altitude.

\section{CONCLUSIONS}

This paper reveals a double-helical structure in the trajectories of interacting agents (particles) subject to mutual 
motion camouflage in three dimensions. Certain modifications of the MMC feedback law lead to behavior that is useful in surveillance tasks.

\section{ACKNOWLEDGEMENTS}

The authors wish to thank Kevin Galloway for valuable discussions.

\section{REFERENCES}

R. L. Bishop. There is more than one way to frame a curve. The American Mathematical Monthly, 82(3):246251, 1975.

K. Ghose, T. Horiuchi, P. S. Krishnaprasad, and C. Moss. Echolocating bats use a nearly time-optimal strategy to intercept prey. PLoS Biology, 4(5):865-873, e108, 2006.

W. D. Hamilton. Geometry for the selfish herd. J. Theor. Biology, 31:295-311, 1971.

E. W. Justh and P. S. Krishnaprasad. Natural frames and interacting particles in three dimensions. Proc. 44th IEEE Conf. Decision and Control, pages 2841-2846, 2005.

E. W. Justh and P. S. Krishnaprasad. Steering laws for motion camouflage. Proc. R. Soc. A, 462:3629-3643, 2006.

M. Mischiati and P. S. Krishnaprasad. Motion camouflage for coverage. Proc. American Control Conference, pages 6429-6435, 2010.

M. Mischiati and P. S. Krishnaprasad. The dynamics of mutual motion camouflage. submitted for publication, 2011.

A. K. Mizutani, J. Chahl, and M. V. Srinivasan. Motion camouflage in dragonflies. Nature, 423:604, 2003.

P. V. Reddy. Steering laws for pursuit. Master's thesis, University of Maryland, College Park, 2007.

P. V. Reddy, E. W. Justh, and P. S. Krishnaprasad. Motion camouflage in three dimensions. Proc. 45th IEEE Conf. Decision and Control, pages 3327-3332, 2006. (see also arXiv:math.OC/0603176).

M. V. Srinivasan and M. Davey. Strategies for active camouflage of motion. Proc. R. Soc. B, 259:19-25, 1995.

E. Wei, E. W. Justh, and P. S. Krishnaprasad. Pursuit and an evolutionary game. Proc. R. Soc. A, 465:1539-1559, 2009 . 